\title{
High precision optical fiber alignment using tube laser bending
}

\author{
K. G. P. Folkersma ${ }^{1}$ - G. R. B. E. Römer ${ }^{1}$ • D. M. Brouwer ${ }^{1}$ • J. L. Herder ${ }^{1}$
}

Received: 24 August 2015 / Accepted: 13 November 2015 / Published online: 30 December 2015

(C) The Author(s) 2015. This article is published with open access at Springerlink.com

\begin{abstract}
In this paper, we present a method to align optical fibers within $0.2 \mu \mathrm{m}$ of the optimal position, using tube laser bending and in situ measuring of the coupling efficiency. For near-UV wavelengths, passive alignment of the fibers with respect to the waveguides on photonic integrated circuit chips does not suffice. In prior research, it was shown that permanent position adjustments to an optical fiber by tube laser bending meets the accuracy requirements for this application. This iterative alignment can be done after any assembly steps. A method was developed previously that selects the optimal laser power and laser spot position on the tube, to minimize the number of iterations required to reach the target position. In this paper, that method is extended to the case where the absolute position of the fiber tip cannot be measured. By exploiting the thermal expansion motion at a relatively low laser power, the fiber tip can be moved without permanent deformation (only elastic strain) of the tube. An algorithm has been developed to search for the optimal fiber position, by actively measuring and maximizing the coupling efficiency. This search is performed before each bending step. Experiments have shown that it is possible to align the fiber with an accuracy of $0.2 \mu \mathrm{m}$ using this approach.
\end{abstract}

Keywords Fiber alignment - Laser tube forming · Laser adjustment algorithms $\cdot$ Alignment algorithm

K. G. P. Folkersma

k.g.p.folkersma@utwente.nl

1 Faculty of Engineering Technology, Chair of Applied Laser Technology, University of Twente, Drienerlolaan 5, 7522 NB Enschede, The Netherlands

\section{Introduction}

Recent development in photonic integrated circuits (PIC's) require advances in the optical alignment and assembly of the components. For single-mode fiber coupled optical chips, the fiber alignment and packaging is the most expensive phase in the manufacturing of these devices [14]. Moreover, for small wavelengths in the near-UV range, the mode field diameter is small, resulting in a required lateral alignment accuracy in the order of $0.1 \mu \mathrm{m}$, to obtain an acceptable insertion loss [1]. Passive alignment methods, such as etched V-grooves in glass or silicon, cannot be employed here, due to the geometrical tolerances (most notably the core-cladding concentricity) of commercial available fibers exceeding the alignment requirements [2]. This also implies that fiber array assemblies cannot be aligned simultaneously, since the core-to-core pitch can not be guaranteed to be within the required tolerances.

Therefore, a one-time active alignment per fiber is often used, where the optical coupling efficiency is minimized by sending light through the device and measuring the transmitted power. A hill-climbing algorithm or other optimization method can be used to optimize the transmission while moving and aligning the fiber by, for example, a high-precision motorized stage $[6,13]$. When the optimal position is found, the fiber is fixed to the chip, usually by UV-curing adhesive [2]. However, adhesives are prone to shrinkage during or after the curing process, which causes misalignment after the final bonding step [8]. Therefore, there is a need for alignment methods to (re)align the fiber actively by an actuator integrated in the device. This onetime alignment is done after any manufacturing processes that might disturb the alignment, such as shrinkage of the used adhesives. To achieve this, we propose using a laser forming micro-actuator. 
Stark et al. [11] used laser forming to align multiple fibers with respect to a micro-lens array with a pitch of $2 \mathrm{~mm}$. One actuator consists of three 'legs' in a ' $\mathrm{Y}$ ' shape, where each leg can be shortened by laser forming. A fiber is fixed in the center of each actuator. The authors achieved a minimum lateral step size of $0.2 \mu \mathrm{m}$ of the fiber. However, the heat input to the legs of this actuator requires careful planning of the irradiations to prevent excessive heating of the fiber and adhesives. Zandvoort et al. [12] aligned multiple optical fibers with respect to an optical chip, using laser forming to shorten the four legs in a ' + ' shaped actuator. Multiple actuators were stacked to align an array of fibers individually, each with an accuracy of $0.25 \mu \mathrm{m}$. However, this actuator requires a large base frame of about $30 \mathrm{~mm} \times 30 \mathrm{~mm}$, which significantly increases the total packaged volume of such a device.

Previous work has shown that laser bending of metal tubes is a feasible method to achieve this one-time precision alignment [4], despite the significant scattering of the bending magnitude and direction of this process. The scattering in bending magnitude was found to increase for increasing laser power (and therefore with increasing bending magnitude). On contrary, the scattering in bending direction was found to decrease with increasing laser power.

Figure 1 shows a sketch of how the tubes and fibers are positioned to the chip. The fibers are fixed in the tubes and the tubes are fixed to the connection block. The connection block can be aligned and joined to the chip, which would typically result in fiber to chip accuracies in the order of 2 to $5 \mu \mathrm{m}$. This assembly allows the use of laser bending to align the individual fibers precisely with respect to the waveguides in the chip, with a waveguide pitch of $1 \mathrm{~mm}$.

Little research is available on the laser bending of tubes with a diameter in the order of $1 \mathrm{~mm}$. Jamil et al. [7] recently studied the bending of nickel micro tubes with an outer diameter of $1 \mathrm{~mm}$ and a wall thickness of $50 \mu \mathrm{m}$,

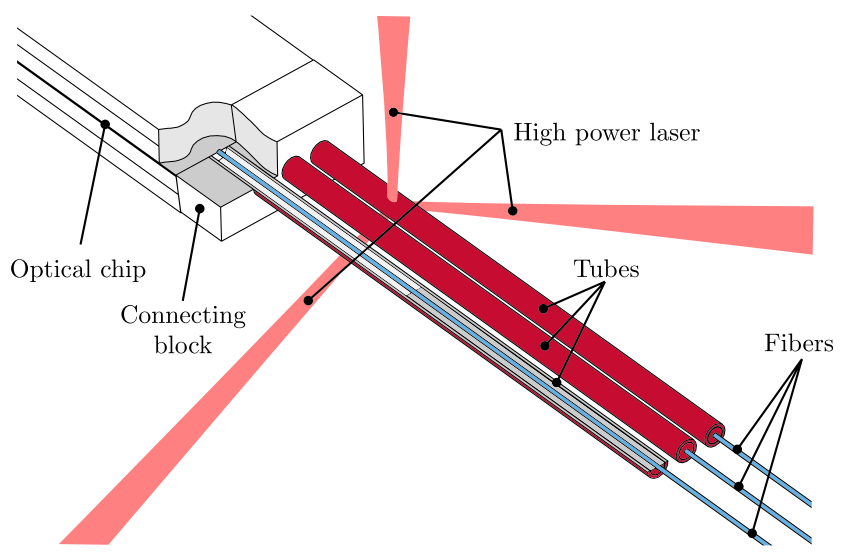

Fig. 1 Sketch of how multiple fibers can be aligned to a PIC using tube bending using a high-power diode laser. The authors concluded that the bending angle is small for any combination of pulse duration and laser power due to the thin walls not offering enough resistance to counteract the thermal expansion, resulting in little plastic deformation. Pre-stressing the tube by displacing its free end resulted in a significant increase of the bending angle, almost linear with the amount of initial displacement. Chandan et al. [3] reported on the laser bending of stainless steel micro tubes with an outer diameter of about $1 \mathrm{~mm}$ and a wall thickness of $150 \mu \mathrm{m}$. The authors concluded that the bending angle increases both with an increasing pulse energy (at constant pulse duration) and with a decreasing pulse duration (at constant pulse energy). Qi and Namba studied the laser bending of $8 \mathrm{~mm}$ 304 stainless steel rods [10], by scanning the laser in the axial direction. The authors achieved a repeatable bending angle of $1.75 \times 10^{-3} \mathrm{mrad}$. Moreover, it was observed that multiple scans increase the bending angle linearly, except for the first scan, which shows a significant larger bending.

\section{Goal and outline}

This paper aims at developing a tube laser bending algorithm to align an optical fiber to a PIC, making use of only the light through the fiber for position sensing. Due to the fiber being fully enclosed by the tube, there is no knowledge of the absolute fiber tip position. However, by moving the fiber, the coupling efficiency can be maximized by actively measuring the transmitted power. The complete iteration scheme for aligning one fiber to its target can be broken down as:

1. Scan. Estimate the relative target position by moving the fiber using a scanning algorithm.

2. Parameter selection. Determine optimal laser power and beam position on the tube to reach the estimated target position.

3. Bending. Execute the laser bending step and measure the coupling efficiency.

4. Stop condition. Evaluate the stop condition based on the coupling efficiency. If it is not met, go to step 1.

The following sections explain these steps in more detail. First, the fiber alignment tube actuator that was previously developed is detailed in Section 3. The proposed scan algorithm as well as the thermal expansion motion is presented in Section 4. In Section 5, the selection of the laser power and laser spot position to reach the scanned target is explained. The scan and bending step are repeated until the stop condition given in Section 6 is met. The algorithms are tested with an experimental setup, see Section 7. The results of experiments with the scan algorithm and 
alignment experiments are given in Sections 8.1 and 8.2, respectively. Finally, the results are discussed in Section 9 and the conclusions are summarized in Section 10.

\section{Fiber alignment by laser tube bending}

The assembly of the tube and the optical fiber is shown in Fig. 2. The single-mode optical fiber is fixed concentrically in the 18-mm-long metal tube using a 10-mm-long mating tube. The laser spot is located at an axial distance $d$ from the fiber tip, at an angle $\phi_{\text {exp. }}$. The steel tube has a outer diameter of $711 \mu \mathrm{m}$ and a wall thickness of $89 \mu \mathrm{m}$. These dimensions were found to perform the best with the alignment algorithm [5]. The bending angles are small, therefore the lateral displacement of the fiber tip can be expressed as $\delta_{\mathrm{r}}=d \cdot \alpha$, where $\alpha$ is the bending angle after the tube has cooled down (see Fig. 2).

\section{Scanning for the target position}

The optimal position of the fiber is where the transmitted power from the fiber into the PIC is maximized. The relative coupling efficiency can be measured by measuring the laser power transmitted through the chip, while a fixed-power laser source is connected to the fiber.

When using the Gaussian beam approximation [9] and assuming the angular alignment is perfect and the axial separation is zero, the theoretical coupling efficiency of the interconnect reads:

$\eta=\left(\frac{2 w_{\mathrm{f}} w_{\mathrm{c}}}{w_{\mathrm{f}}^{2}+w_{\mathrm{c}}^{2}}\right)^{2} \exp \left(-\frac{2 \delta^{2}}{w_{\mathrm{f}}^{2}+w_{\mathrm{c}}^{2}}\right)$,
Where $\delta$ is the lateral offset between the fiber and target, and $w_{\mathrm{f}}$ and $w_{\mathrm{c}}$ are the mode-field radii of the fiber and chip, respectively. Figure 3 shows the coupling efficiency for $w_{\mathrm{f}}=1.55 \mu \mathrm{m}$ and $w_{\mathrm{c}}=2.25 \mu \mathrm{m}$, which is used in the remainder of this paper. The fact that the optimal coupling efficiency is not 1 is due to the mode mismatching of the fiber and the chip. As can be concluded from the figure, for $\delta=0.2 \mu \mathrm{m}$, the loss is $1 \%$ of the optimal coupling efficiency. When $\delta$ is larger than $4 \mu \mathrm{m}$, the coupling efficiency is close to zero, and cannot be reliably measured.

\subsection{Thermal expansion motion}

The coupling efficiency through the interconnect is the only measured quantity for the two-dimensional alignment. Therefore, a searching method for the optimal alignment position is required. This searching can be done between each bending step, by exploiting the thermal expansion motion when heating the tube by laser irradiation.

That is, at low laser power ( 1 to $3 \mathrm{~W}$ ) and short pulse duration $(50 \mathrm{~ms})$, the yield stress in the tube is not exceeded, and only thermal and elastic strain occurs. That means that the final bending angle after cooling down is not changed. The direction of thermal expansion is assumed to be equal to the laser spot angle around the tube $\left(\phi_{\exp }\right)$, and is therefore known. The magnitude of the thermal expansion bending angle $\beta$, however, is not known.

Using the experimental setup (described in Section 7), it has been found that the bending due to thermal expansion reproduces very well. A prerequisite for this repeatability is that no initial stresses are present in the tube near the laser spot, and no oxidation of the tube surface due to the laser heat is formed. Therefore, the bending angle due to thermal expansion is characterized at $d=7.5 \mathrm{~mm}$, which

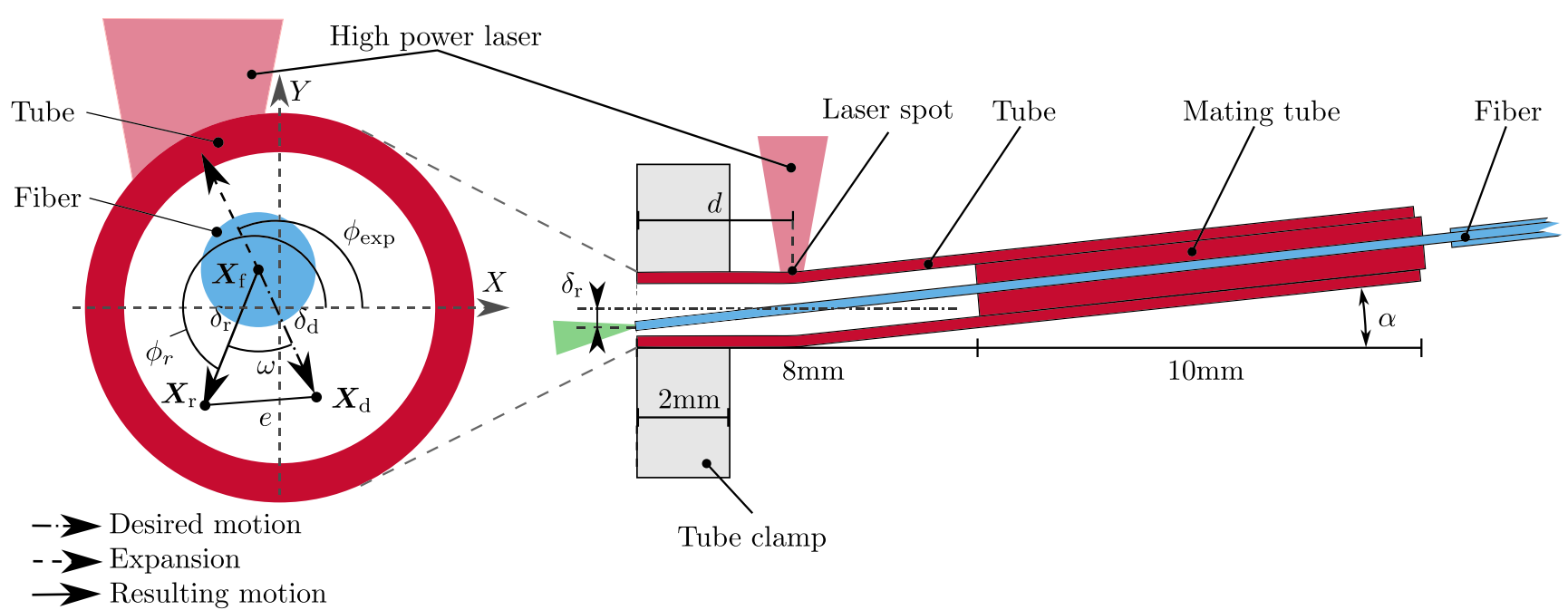

Fig. 2 Fiber and tube assembly used in the experiments. The coordinates of the fiber tip are depicted on the left 
is outside the region where the actual bending is to occur ( $3 \mathrm{~mm} \leq d \leq 7 \mathrm{~mm}$ ). Figure 4 shows the measurement of $\beta$ for 36 laser pulses on different locations on four identical tubes, all at a laser power of $3 \mathrm{~W}$. The repeatability of this expansion angle $\beta$ is $0.15 \mathrm{mrad}$, which corresponds with a repeatability of $1.1 \mu \mathrm{m}$ at the fiber tip. The maximum bending angle at the end of the pulse is $\beta_{\max }=3.2 \mathrm{mrad}$, corresponding to a tip displacement of $24 \mu \mathrm{m}$. This limits area in which the target can be found to be a circle with a radius of $24 \mu \mathrm{m}$ around the current position $\boldsymbol{X}_{\mathrm{f}}$ (see Fig. 2). The same measurements have been repeated for a laser power of 2 and $1.5 \mathrm{~W}$. The repeatability of the expansion angle were found to equal 0.12 and $0.10 \mathrm{mrad}$, respectively, corresponding with a fiber tip position repeatability of 0.9 and $0.75 \mu \mathrm{m}$.

To estimate $\beta$ when aligning the fiber to a chip (where the fiber tip position is unknown), the heating phase of these measurements is fitted with a fourth-order polynomial $\bar{\beta}$, shown by the red dashed line in Fig. 4. This curve relates the elapsed time $t$ of the laser pulse to the expansion angle. With the known scan direction $\phi_{\text {scan }}=\phi_{\text {exp }}$, the position of the fiber tip $\boldsymbol{X}_{\mathrm{f}}$ during heating can be estimated by:

$\hat{X}_{\mathrm{f}}(t)=d \cdot \bar{\beta}(t)\left[\begin{array}{c}\cos \phi_{\text {scan }} \\ \sin \phi_{\text {scan }}\end{array}\right]$.

\subsection{Scanning algorithm}

The scanning algorithm is an iterative algorithm that aims at finding the maximum coupling efficiency, by moving the fiber with respect to the target. The location of this maximum, relative to the current position, then defines the direction and magnitude for next tube bending step. Figure 5 shows the flowchart of the scanning algorithm.

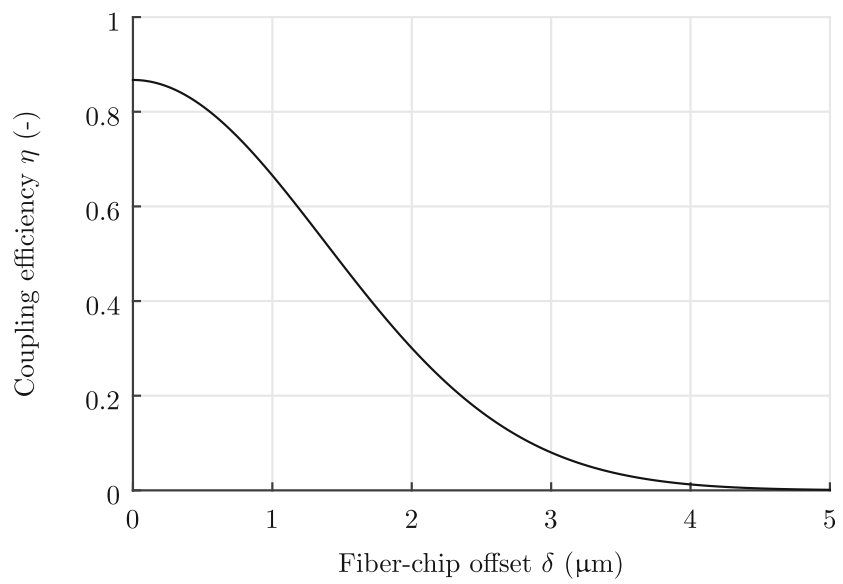

Fig. 3 Theoretical coupling efficiency of a fiber to chip coupling for $w_{\mathrm{f}}=1.55 \mu \mathrm{m}$ and $w_{\mathrm{c}}=2.25 \mu \mathrm{m}$, assuming perfect tilt and axial alignment
For each scanning iteration $i$, the fiber is moved by thermal expansion in the direction $\phi_{\text {scan }}^{(i)}$. The initial direction $\phi_{\text {scan }}^{(1)}$ is set to the direction of the initial guess of the relative target position $\hat{\boldsymbol{X}}_{\mathrm{d}}^{(0)}$. For the first scan, $\hat{\boldsymbol{X}}_{\mathrm{d}}^{(0)}$ is set by the user. For each subsequent scan, $\hat{\boldsymbol{X}}_{\mathrm{d}}^{(0)}$ is set to the estimated destination position from the previous scanning action.

The relative coupling efficiency $\eta$ is measured in real time during the scan time $0 \leq t \leq t_{\text {pulse. }}$. If no signal is found (i.e., $\max \eta(t)=0$ ), or if the number of scan iterations is less than four, the next scan direction $\phi_{\text {scan }}$ is set in the center of the largest 'unscanned' area, and the measurement is repeated. As a result, the four initial scan iterations always result in a ' + ' shape of the scan path, see Fig. 6. This ensures that an inaccurate initial target guess does not result in an excessive amount of iterations for the hill climbing algorithm.

When a signal is found for the current iteration, the maximum coupling efficiency $\eta_{\max }^{(i)}$ occurs at $t=t_{\max }$. The best estimate of $\boldsymbol{X}_{\mathrm{d}}$ is now $\hat{\boldsymbol{X}}_{\mathrm{d}}^{(i)}=\hat{\boldsymbol{X}}_{\mathrm{f}}\left(t_{\max }\right)$. The next scan direction is now chosen according to a simple hill-climbing algorithm by

$\phi_{\text {step }}^{(i+1)}=-0.5 \phi_{\text {step }}^{(i)}$

$\phi_{\text {scan }}^{(i+1)}=\phi_{\text {scan }}^{\left(i_{\text {best }}\right)}+\phi_{\text {step }}^{(i+1)}$,

where $i_{\text {best }}$ is the scan iteration where the overall maximum coupling efficiency occurred. This means that the next scan direction alternates around the direction where the best coupling efficiency was measured, with the step size halving each iteration.

If a signal is found $(\eta(t)>0)$ for four or more scan iterations, a two-dimensional Gaussian surface is fitted through

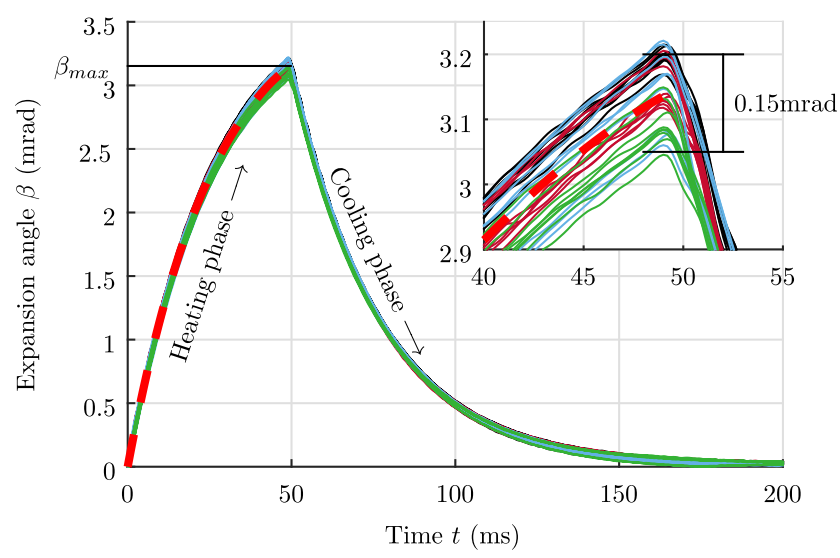

Fig. 4 Measured bending angle for 36 laser pulses at different positions on four identical tubes (each tube is indicated by a separate color). For the pulse duration of $50 \mathrm{~ms}$ and laser power of $3 \mathrm{~W}$, the yield stress is not exceeded, and therefore the final deformation angle is zero. The dashed red line indicates the fourth-order polynomial fit through the heating phase, used for estimating the angle for thermal expansion 
the measurement data ( $\eta$ and $\hat{X}_{\mathrm{f}}$ ) of all previous iterations, see Fig. 6. If the squared 2-norm of the residuals of this fit is above 10, it is discarded. This is necessary to prevent bad fits due to numerical problems or noise in the measured data. If the fit is discarded, the hill-climbing is continued. Otherwise, the maximum of this fit is now the best estimate of $\boldsymbol{X}_{\mathrm{d}}$, and $\phi_{\mathrm{scan}}^{(i+1)}$ is set to the direction of this maximum.

The iteration is stopped when the change in coupling efficiency from the previous iteration and the difference with

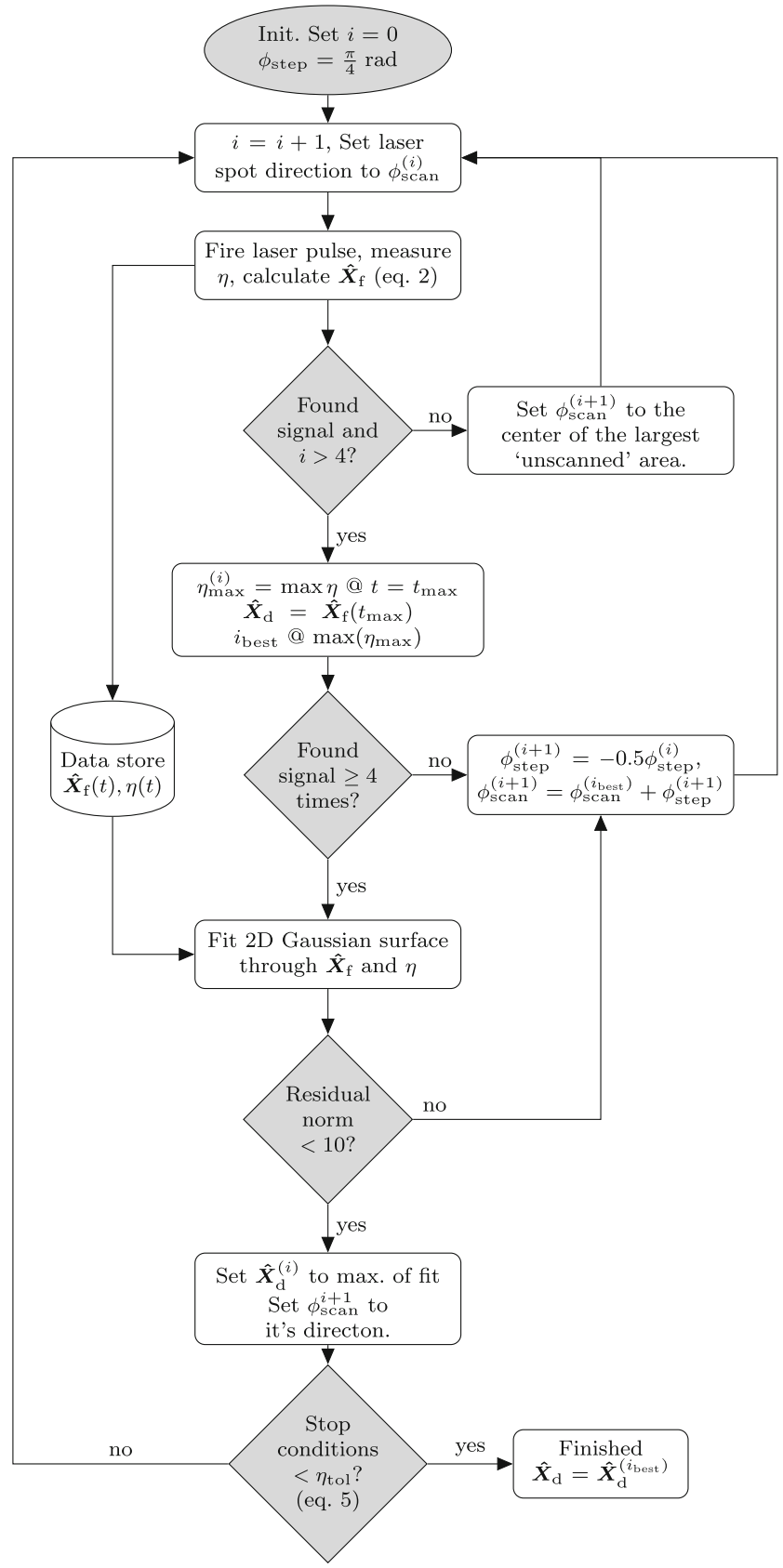

Fig. 5 Flowchart of the scanning algorithm the overall maximum is within a tolerance $\eta_{\text {tol }}$. That is, when both

$$
\begin{aligned}
& \left|\eta_{\max }^{(i)}-\eta_{\max }^{(i-1)}\right|<\eta_{\text {tol }} \text { and } \\
& \left|\eta_{\max }^{(i)}-\eta_{\max }^{\left(i_{\text {best }}\right)}\right|<\eta_{\text {tol }}
\end{aligned}
$$

are satisfied. With decreasing $\eta_{\text {tol }}$, the target position estimation is more accurate, at the cost of more iterations. Therefore, the tolerance depends on the coupling efficiency $\eta_{0}$ before the scan as $\eta_{\text {tol }}=0.1 \cdot\left(1-\eta_{0}\right)$. That is, the tolerance is $10 \%$ of the difference of the initial coupling efficiency to the theoretical maximum. This results in a more accurate estimation of $\boldsymbol{X}_{\mathrm{d}}$ when the target is already close.

Furthermore, the laser power is lowered when the target is near, to increase the accuracy of the fit $\bar{\beta}$ (see Section 4.2). The power is reduced to 2 or $1.5 \mathrm{~W}$ when the estimated distance to the target is below 2 or $1 \mu \mathrm{m}$, respectively.

\section{Laser parameter selection}

The estimated destination location $\hat{\boldsymbol{X}}_{\mathrm{d}}$ is found by scanning before each bending step (see Section 4.2). This position is relative to the current fiber position $\boldsymbol{X}_{\mathrm{f}}$. Therefore, the estimated distance of the fiber tip to the target is $\hat{\delta}_{\mathrm{d}}=\left\|\hat{\boldsymbol{X}}_{\mathrm{d}}\right\|$.

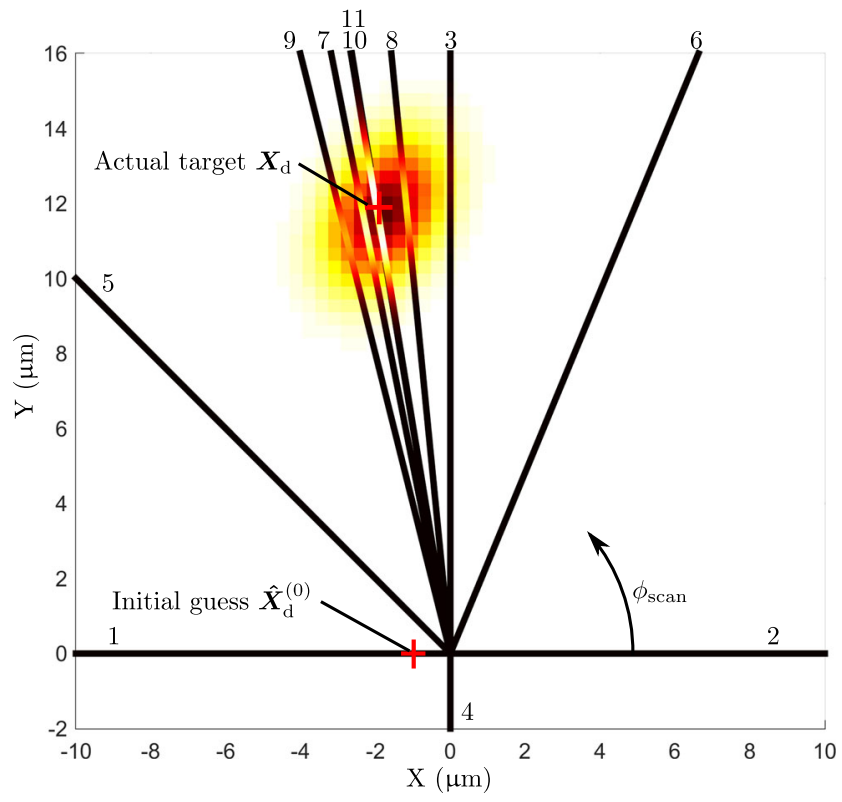

Fig. 6 Example of one target scan, with the rest position in the origin. Each numbered line is a scan iteration using the thermal expansion of the tube. The line color indicates the measured signal (black: no signal, white: high signal), the shaded area indicates the Gaussian surface fit trough these signals (with inverse colors for contrast). Note the ' + ' shape of the first four iterations, even when a signal was already found at iteration 3. At iteration 5, the hill climbing starts, ending at iteration 11 , which is almost equal to iteration 10 , and meets the stop condition 
A method has been developed [5] that determines the optimal laser power $P_{\text {opt }}$ and laser spot position $d_{\text {opt }}$ to minimize the required number of bending steps. This method takes the scattering into account, by 'learning' from position measurements of previous bending steps. A tradeoff has been identified between high certainty in either the bending angle $\alpha$ when choosing a low laser power, or in bending direction $\phi_{\mathrm{r}}$ when choosing a high laser power. An optimum in $d$ and $P$ can be found or each desired step size $\delta_{\mathrm{d}}$, by minimizing the expectation of the error $e$ (see Fig. 2) after the bending step, by assuming a normal probability distribution for $\alpha$ and $\omega$, with a mean and variance depending on the laser power $P$.

However, as stated earlier in this paper, it is assumed that the fiber position cannot be measured directly. Therefore, the learning algorithm from [5] cannot be used. It is therefore required to gather historical response data of the bending angle and direction to the laser power beforehand. Figure 7 shows the optimal parameters used in this paper, based on the measurements presented in [5].

\section{Stop condition of alignment iteration}

The scanning and bending iterations continue until the coupling efficiency $\eta$ is within $1 \%(0.15 \mu \mathrm{m}$, see Eq. 1$)$ of the optimal coupling efficiency $\eta_{\text {opt }}$. However, $\eta_{\text {opt }}$ is unknown beforehand. Therefore, $\eta_{\mathrm{opt}}$ is estimated by the maximum coupling efficiency that has been measured during the past scanning and bending steps. This estimated maximum $\hat{\eta}_{\mathrm{opt}}$ converges to $\eta_{\text {opt }}$ with an increasing number of bending and scanning steps. The stop condition is defined by

$1-\frac{\eta}{\hat{\eta}_{\mathrm{opt}}}<0.01$

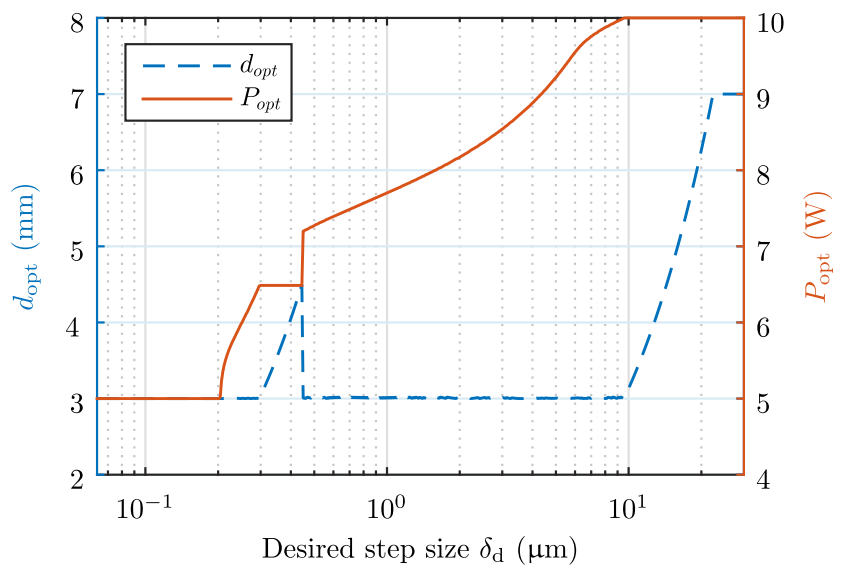

Fig. 7 The optimal parameters depending on the desired fiber tip displacement $\delta_{\mathrm{d}}$, based on the optimization and data from [5]. The laser power is limited to $10 \mathrm{~W}$ to prevent melting of the tube
However, the stop condition can be met before $\hat{\eta}_{\text {opt }}$ has converged close to $\eta_{\mathrm{opt}}$. This is avoided by requiring the condition in Eq. 6 is met twice. The scan step in between those two bending steps (see Section 4.2) ensures that the estimate $\hat{\eta}_{\text {opt }}$ is improved, due to the scanning in all directions.

\section{Experimental setup}

To test the performance of the scanning and alignment algorithms, an experimental setup has been developed that allows for fully unattended laser bending of the tube to align an optical fiber to a pre-set destination position.

The position of the fiber cannot be measured during the fiber to chip alignment. However, the lateral $(\mathrm{X}, \mathrm{Y})$ displacement of the fiber tip is of interest to evaluate the performance of the algorithm. Therefore, this experimental setup is used to measure the fiber tip position by the light emitted from the fiber, instead of the coupling efficiency. This means that a PIC chip is not in place in this setup. However, the coupling efficiency with respect to such a chip is simulated in real-time by using Eq. 1 and the measured distance to the destination $\delta_{\mathrm{d}}$. This simulated coupling efficiency is therefore used to test the alignment algorithm.

\subsection{Tube sample and fixation}

The 304 stainless steel tubes received a hard temper treatment after drawing. The tube dimensions are listed in Table 1. A single-mode fiber (Thorlabs SM600) was fixed to each tube as shown in Fig. 2. The tube with the fiber is clamped in a custom brass clamp over 2-mm length (see Figs. 8 and 2). The clamp with the tube can be aligned to the measurement system in all directions (except for the rotation around the tube axis) using motorized and manual stages.

Table 1 Summary of experimental parameters

\begin{tabular}{ll}
\hline Property & Value \\
\hline Laser power & $5-10 \mathrm{~W}$ (bending) \\
Laser $1 / e^{2}$ spot diameter & $1-3 \mathrm{~W}$ (scanning) \\
Laser wavelength & $400 \mu \mathrm{m}$ \\
Laser pulse duration & $1080 \mathrm{~nm}$ \\
Tube outer diameter & $100 \mathrm{~ms}$ \\
Tube wall thickness & $711 \mu \mathrm{m}$ \\
Tube material & $89 \mu \mathrm{m}$ \\
Fiber diameter & $304 \mathrm{Stainless}$ steel \\
\hline
\end{tabular}




\subsection{Laser and beam delivery}

A fiber laser (JK 100FL, max. $100 \mathrm{~W}, 1080 \mathrm{~nm}$ ) showing a Gaussian intensity distribution and a $1 / e^{2}$ spot diameter of $400 \mu \mathrm{m}$ at the tube surface, is used to heat and deform the tubes. The laser spot is either positioned directly on the tube, or via one of two fixed mirrors near the tube, see Fig. 9. A camera is mounted on the focusing head to align the laser beam to the tube. Using the tip/tilt mirror, three radial positions spaced $120^{\circ}$ from each other, and the complete tube length are accessible by the laser spot. Additionally, since the spot size is smaller than the tube diameter, a small deviation of $\pm 35^{\circ}$ from these radial positions can be tolerated. This means that for $\phi_{\text {exp }}$ three evenly spaced 'blind spots' of $50^{\circ}$ are present that can not be reached by the laser beam.

\subsection{Fiber position measurement}

Figure 9 shows the optical elements to measure the displacement of the fiber tip; Fig. 8 shows a photo of the tube with fiber positioned in front of the measurement system. A low power laser source $(2 \mathrm{~mW}, 650 \mathrm{~nm})$ was connected to the free end of the fiber. The beam emitted from the fiber was focused with an aspheric lens with a focal length of $4.6 \mathrm{~mm}$. The beam position was measured by two

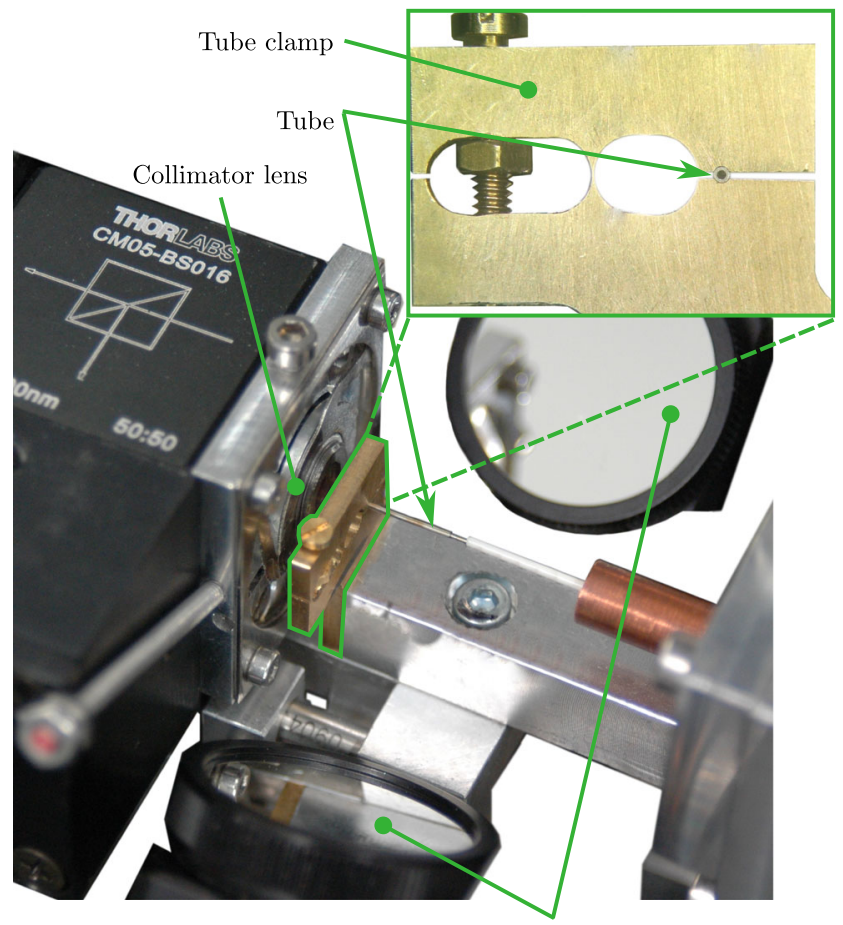

Fixed mirrors

Fig. 8 Detail photo of the experimental setup duo-lateral position sensing detectors (PSDs) via beamsplitters. Furthermore, a camera is used to find the focus position of the fiber with respect to the lens, see Fig. 9.

To calibrate the PSD signals with respect to the lateral displacement, the relative position of the clamp with the tube and fiber with respect the PSDs is measured by capacitive displacement sensors. The relation of the signal from the PSDs and the fiber position is calibrated by moving the clamp with the tube, and measuring the translation with the capacitive sensors. The (quadratic) relation was found by multivariate linear regression, using the least squares estimate.

After calibration, the capacitive sensors are used to subtract any external influences (for example vibrations or thermal variations) from the measurement. The resulting signal gives the lateral position of the fiber tip with a resolution of $50 \mathrm{~nm}$, a maximum absolute measurement error of $0.2 \mu \mathrm{m}$ and a repeatability better than $0.1 \mu \mathrm{m}$ over a range of $\pm 25 \mu \mathrm{m}$.

All measurement signals are processed by a Matlab Simulink Real-time environment, sampling at $5 \mathrm{kHz}$. For each deformation step, the signals are processed to extract the relative tube deformation magnitude and direction. The



Fig. 9 Schematic overview of the experimental setup 
Table 2 The results of five alignment experiments. All experiments start at the location $\boldsymbol{X}_{\mathrm{f}}=(0,0)$

\begin{tabular}{llll}
\hline$\#$ & Target $\boldsymbol{X}_{\mathrm{d}}(\mu \mathrm{m})$ & Nr. of steps & Final error $\boldsymbol{e}(\mu \mathrm{m})$ \\
\hline 1 & $(-8,6)$ & 16 & 0.19 \\
2 & $(1,10)$ & 8 & 0.04 \\
3 & $(-6,-8)$ & 12 & 0.19 \\
4 & $(5,9)$ & 5 & 0.09 \\
5 & $(-95)$ & 12 & 0.09 \\
\hline
\end{tabular}

beam position, laser power, triggering, and tube positioning stages are all fully computer-controlled.

The setup and measurement system is explained in more detail in [4].

\section{Results}

Both the scanning and alignment algorithms were tested with the experimental setup. Five alignment experiments are performed, where the initial distance to the target was set to $10 \mu \mathrm{m}$, each with a random direction, see Table 2 .

\subsection{Target searching experiments}

The scan algorithm has been evaluated with the experiments listed in Table 2. The estimated target position error is defined as $e_{\text {scan }}=\left|\hat{\boldsymbol{X}}_{\mathrm{d}}-\boldsymbol{X}_{\mathrm{d}}\right|$. Figure 10 shows the histogram of the error relative to the distance to the target $\left(e_{\text {scan }} / \delta_{\mathrm{d}}\right)$ for 94 scan experiments. The histogram shows that $90 \%$ of the scans have an error that is smaller than the distance to the target, resulting in a bending step that is likely to converge to the target. The remaining $10 \%$ of the scans

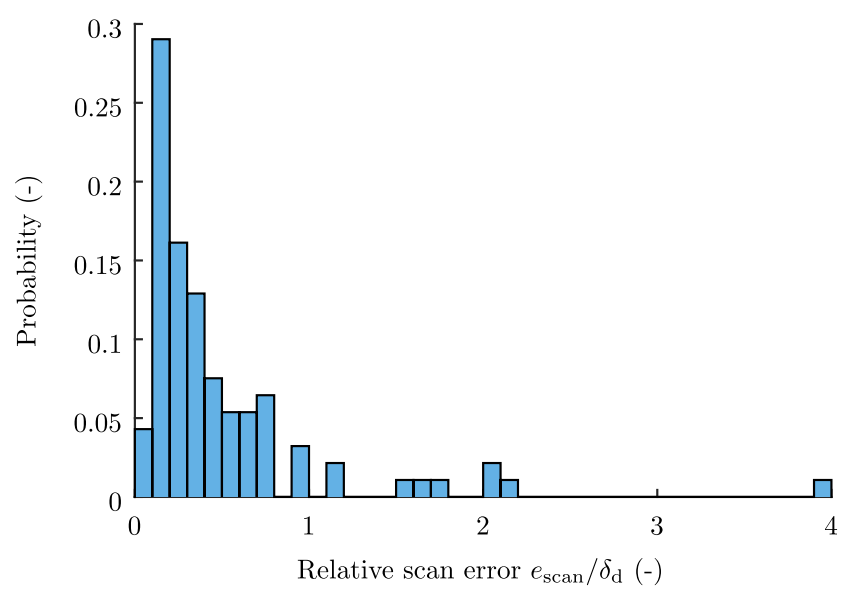

Fig. 10 Histogram of the relative error of the scanning algorithm to the (real) distance to the target $\delta_{\mathrm{d}}$ for 94 scan experiments. $e_{\text {scan }}=$ $\left|\hat{\boldsymbol{X}}_{\mathrm{d}}-\boldsymbol{X}_{\mathrm{d}}\right|$ have an error larger than the distance to the target, which mostly happens when the $\delta_{\mathrm{d}}<0.5 \mu \mathrm{m}$. This is a limitation on the accuracy of the fit $\bar{\beta}$ at the start of the pulse (see Section 4.1). This accuracy is mostly limited by the sampling time of the acquisition hardware combined with the fast motion of the fiber during the thermal expansion. An even lower laser power would result in a slower expansion, but $1.5 \mathrm{~W}$ is the minimum power for the used laser system.

\subsection{Alignment experiments}

The alignment algorithm has been tested using the estimated target positions $\hat{\boldsymbol{X}}_{\mathrm{d}}$ (see Section 8.1). For experiments listed in Table 2, the number of steps required to reach the stop condition is between 5 and 16. All experiments ended within $0.2 \mu \mathrm{m}$ from the target position. A typical path is

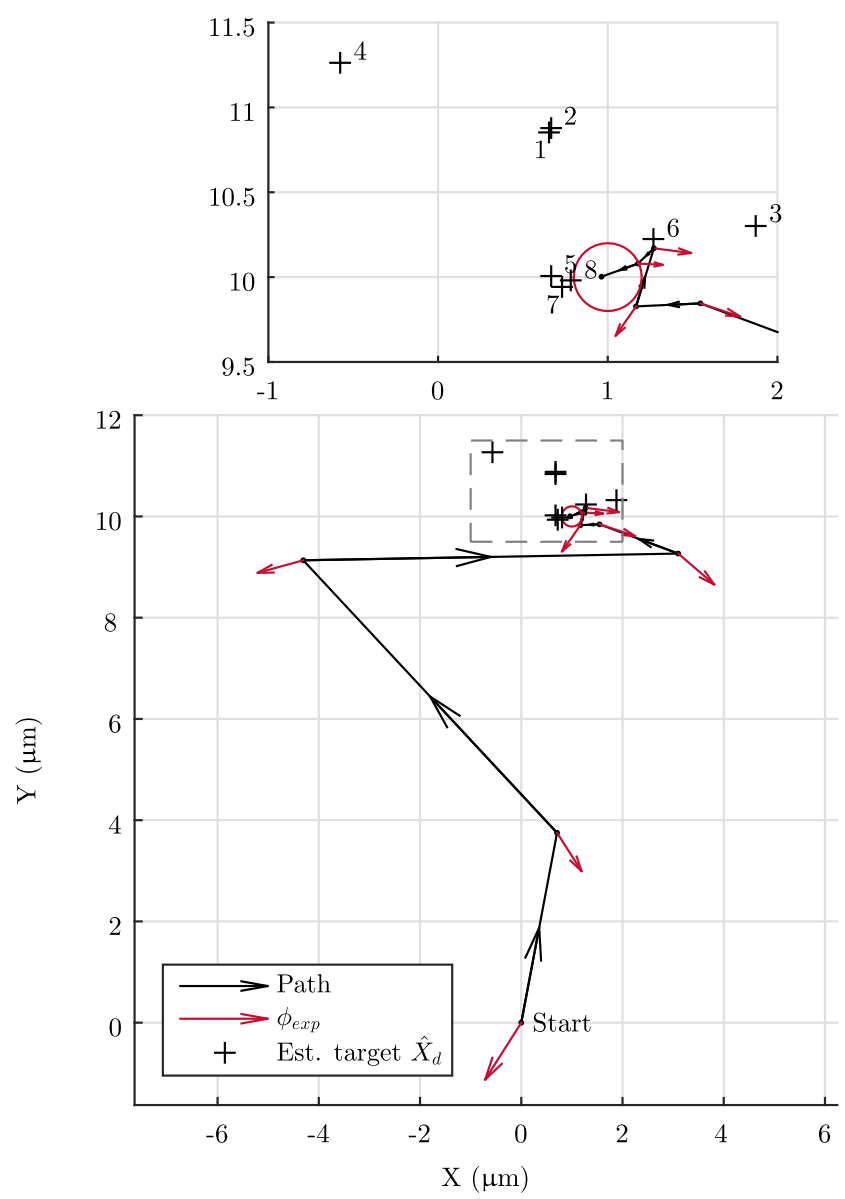

Fig. 11 Path of alignment experiment 2 (see Table 2). The red circle indicates the actual target zone. The black line indicates the location of the fiber tip between each deformation step, connected by straight lines. The red arrows indicate the measured direction of the thermal expansion at each bending step. The scanned estimated destination $\hat{\boldsymbol{X}}_{\mathrm{d}}$ is indicated by a numbered ' + ', corresponding to the scan prior to each bending step 
shown in Fig. 11. Note the large deviation from the planned path for the second step in this figure. This is due to the 'blind spots' on the tube that cannot be accessed by the laser (see Section 7), instead the closest accessible direction is chosen.

\section{Discussion}

In this paper, we have shown the principle of precision optical fiber alignment by laser tube forming on steel tubes. It is expected that even better accuracy and stability can be obtained using tubes from low thermal expansion metals such as Invar. Unlike steel, the linear thermal expansion coefficient (CTE) of Invar at room temperature $\left(1.6 \times 10^{-6} \mathrm{~K}^{-1}\right)$ is comparable to that of the chip $\left(1 \times 10^{-6} \mathrm{~K}^{-1}\right.$ to $\left.2.5 \times 10^{-6} \mathrm{~K}^{-1}\right)$. Above $200^{\circ} \mathrm{C}$, the CTE of Invar rises sharply, making it suitable for laser forming, while the position is stable at the operating temperature of the device.

Furthermore, the length of the tube has been chosen such that $d$ can be chosen between 3 and $7 \mathrm{~mm}$, allowing for a wide range of step sizes $\delta_{\mathrm{d}}$. However, it is expected that the initial 'rough' alignment of the tube assembly is within $5 \mu \mathrm{m}$. Figure 7 shows that in this case $d_{\text {opt }}$ does not exceed $4.5 \mathrm{~mm}$. Therefore, the tube (and the free fiber length) can be shortened significantly, to increase the stiffness of fiber tip position and reduce its mass. This improves the stability of the fiber tip when the device is subject to external vibrations.

\section{Conclusion}

Fiber positioning using micro tube laser bending was demonstrated to be accurate enough for the alignment with respect to an photonic integrated circuit chip. Because the fiber position cannot be measured inside the tube, the optical coupling efficiency is measured instead. The maximum of this coupling efficiency is found by moving the fiber tip, by exploiting the thermal expansion motion of the tube. A scanning algorithm is developed to find the estimated position of this maximum, even when no light is transmitted in the initial position. The estimated position is used as target for the laser forming step, resulting in a permanent deformation. This is repeated until a stop condition based on the coupling efficiency is met.

Experiments using this searching and alignment algorithm show that a final accuracy of $0.2 \mu \mathrm{m}$ is achieved within 5 to 16 steps. The accuracy is limited by the repeatability of the thermal expansion motion and the measurement timing precision, while scanning for the target position.
Acknowledgments This research is financially supported by the Dutch association Innovatiegerichte OnderzoeksProgrammas (IOP) Photonic Devices (IPD100014), part of the Ministry of Economic Affairs.

Open Access This article is distributed under the terms of the Creative Commons Attribution 4.0 International License (http:// creativecommons.org/licenses/by/4.0/), which permits unrestricted use, distribution, and reproduction in any medium, provided you give appropriate credit to the original author(s) and the source, provide a link to the Creative Commons license, and indicate if changes were made.

\section{References}

1. Böttger G, Schröder H, Jordan R (2013) Active or passive fiber-chip-alignment: approaches to efficient solutions. In: Optoelectronic Interconnects XIII, vol 8630, pp 863,006-863,006-17. doi:10.1117/12.2014176

2. Boudreau RABSM (2005) Passive Micro-Optical Alignment Methods, vol 1. CRC Press, Boca Raton

3. Chandan K, Saha P, Mishra PK (2005) Laser bending of micro tubes. In: Innovating The Future Through Manufacturing, p 227. Alpha Science Int. Ltd. doi:10.1080/00401706.1969.10490657

4. Folkersma KGP, Brouwer DM, Römer GRBE (2015) Micro tube laser forming for precision component alignment. Submitted

5. Folkersma KGP, Brouwer DM, Römer GRBE (2015) Robust precision alignment algorithm for micro tube laser forming. Submitted

6. Gabler C, Li K, Hackwood S, Beni G (1987) An optical alignment robot system. In: Proceedings of the SPIE Integration and Packaging of Optoelectronic Devices, vol 0703, pp 8-28. doi:10.1117/ 12.965184

7. Jamil MC, Fauzi EI, Juinn C, Sheikh M (2015) Laser bending of pre-stressed thin-walled nickel micro-tubes. Opt Laser Technol 73(0):105-117. doi:10.1016/j.optlastec.2015.04.012

8. Lin Y, Liu W, Shi F (2006) Adhesive joint design for minimizing fiber alignment shift during UV curing. IEEE Trans Adv Packag 29(3):520-524. doi:10.1109/TADVP.2005.850505

9. Marcuse D (1977) Loss analysis of single-mode fiber splices. Bell System Technical Journal 56(5):703-718. doi:10.1002/j.15387305.1977.tb00534.x

10. Qi L, Namba Y (2011) Precision laser adjustment using \{CW\} diode laser. Precis Eng 35(1):126-132. doi:10.1016/j.precisioneng.2010.08.002

11. Stark M, Esser G, Lamott A, Geiger M (2004) Laser-based microalignment for fabrication of highly precise 2D fiber collimator arrays. In: Photon Processing in Microelectronics and Photonics III, vol 5339, pp 144-155. SPIE. doi:10.1117/12.529072

12. van Zantvoort J, Plukker S, Dekkers E, Petkov G, Khoe G, Koonen A, de Waardt H (2006) Lensed fiber-array assembly with individual fiber fine positioning in the submicrometer range. IEEE Journal of Selected Topics in Quantum Electronics 12(5):931-939. doi:10.1109/JSTQE.2006.882633

13. Zhang R, Shi F (2004) A novel algorithm for fiber-optic alignment automation. IEEE Trans Adv Packag 27(1):173-178. doi:10.1109/TADVP.2004.825434

14. Zhou H, Liu W, Lin Y, Mondal S, Shi F (2002) A novel assembling technique for fiber collimator arrays using UV-curable adhesives. IEEE Trans Adv Packag 25(4):481-487. doi:10.1109/ TADVP.2002.807602 